\title{
Optimal cantilever dynamic vibration absorbers by Timoshenko Beam Theory
}

\author{
M. Cavacece ${ }^{\mathrm{a}, *}$ and L. Vita ${ }^{\mathrm{b}}$ \\ ${ }^{a}$ Department of Structural Engineering, University of Cassino, 03043 Cassino, Italy \\ bepartment of Mechanical Engineering, University of Rome “Tor Vergata”, 00133 Rome, Italy \\ Professor Bruno Piombo has shown us that the passion for the research and for teaching is a never-ending gift.
}

\begin{abstract}
A double-ended cantilever beam as a distributed parameter dynamic vibration absorber has been applied to a singledegree-of-freedom system subjected to harmonic forces.

In this investigation, the beam has been analyzed under the well known model of Timoshenko and the computation of best parameters is based on the Chebyshev's optimality criterion.

This is somewhat novel in the field since:

- the design of cantilever beams as dynamic vibration absorbers is usually made under the hypotheses of the Euler-Bernoulli theory;

- it is the first time that the Chebyshev's criterion is applied to the design of a double-ended cantilever beam used as a dynamic vibration absorber.
\end{abstract}

For a ready use of the results herein presented, design charts allow a quick choice of optimal parameters such as tuning ratio and mass ratio.

\section{Introduction}

The classical dynamic vibrations absorber is made up of two masses. The first one is subjected to an harmonic load which lead to a vibrational motion of this mass, the second mass is connected to the main mass by means of a spring element. Thus choosing properly the weight of the second mass and the spring stiffness, the vibration amplitude of the main mass could be reduced and, under ideal conditions, also cancelled $[4,12]$.

In fact when an absorbing mass-spring system is attached to the main mass and the resonance of the absorber is tuned to match that of the main mass, the motion of the main mass is reduced to zero at its resonance frequency (Fig. 1).

The test case proposed in this paper concerns a double-ended cantilever beam used as a dynamic vibration absorber [8]. As a consequence the parameters to be set in order to reduce the vibrations of the main mass are the intrinsic elasticity of the beam and its weight. For a more faithful modeling of the beam behavior, the authors have deduced the dynamic equations of the system under analysis by means of the Timoshenko's model [7]. These equations have been used under the conditions set by the Chebyshev's theorem in order to define the optimal features of the beam (e.g. cross section area, length, thickness) [9]. The results have been compared with the ones obtained following the Euler - Bernoulli's model of the beam as reported by Jacquot and Foster [8].

\footnotetext{
*Corresponding author. E-mail: cavacece@unicas.it.
} 

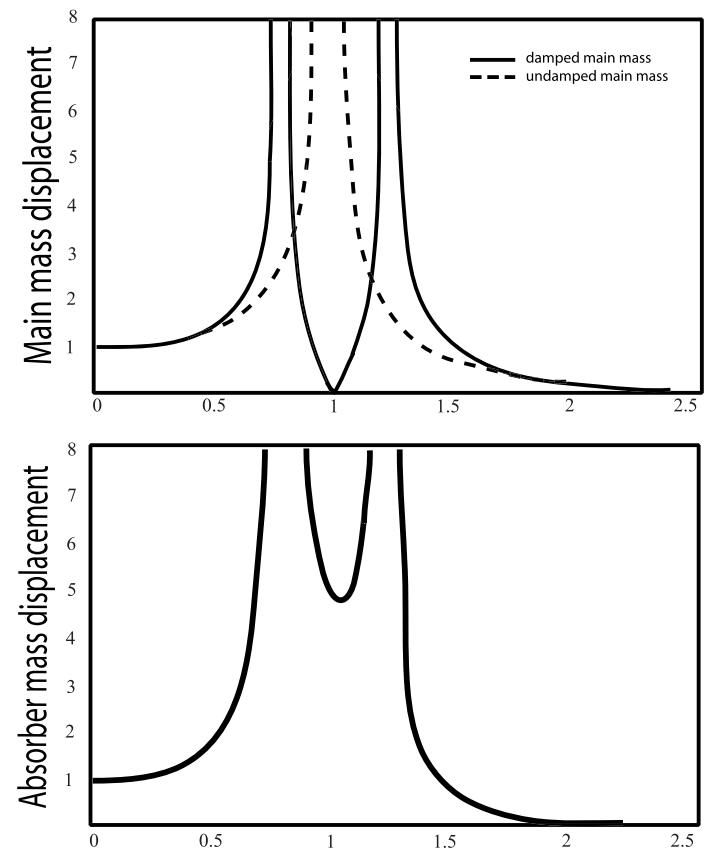

Fig. 1. Plots of the mass displacements.

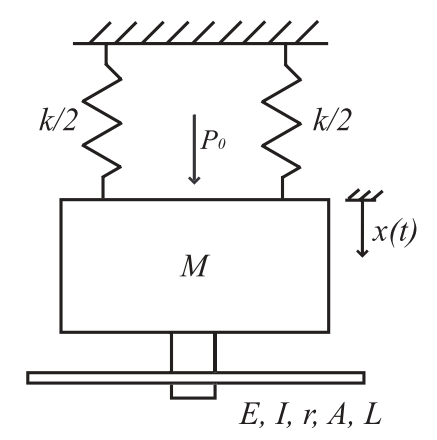

Fig. 2. Scheme of the dynamic vibrations absorber.

\section{System modeling}

The undamped system, shown in Fig. 2, is composed of a spring-supported lumped mass which is free to move only vertically.

Attached to the mass there is the double-ended cantilever beam as shown. Separating the system into three parts as shown in Fig. 3, the method of superposition is applied to examine the coupling between the subsystems.

The equation of motion for the main mass $M$ is

$$
M \frac{\delta^{2} w}{\delta t^{2}}+k w=P_{0} e^{j \omega t}+2 V_{0} e^{j \omega t}
$$

where the $V_{0} e^{j \omega t}$ term is the yet unknown vibration absorber force for a single beam on the main mass. This force can be obtained as the shear force at the root of a displacement-excited cantilever beam. The governing equation for the cantilever beam, under the assumption of Timoshenko bending theory, is

$$
E I_{n} \frac{\delta^{4} y}{\delta x^{4}}+A \rho \frac{\delta^{2} y}{\delta t^{2}}-\rho I_{n}\left(1+\frac{E}{\chi G}\right) \frac{\delta^{4} y}{\delta x^{2} \delta t^{2}}+\frac{\rho^{2} I_{n}}{\chi G} \frac{\delta^{4} y}{\delta t^{4}}=0
$$




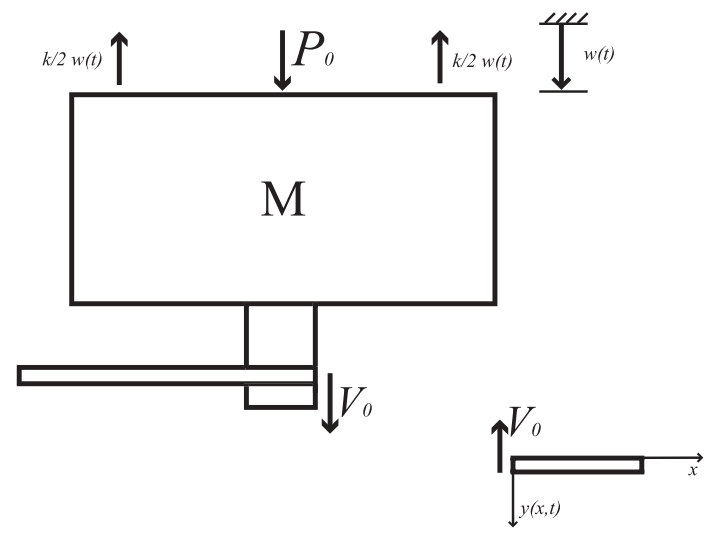

Fig. 3. Freebody diagram of the system.

with the following boundary conditions:

$$
\begin{aligned}
& y(0, t)=W_{0} e^{j \omega t} \\
& \varphi(0, t)=0 \\
& \frac{\delta \varphi(L, t)}{\delta x}=0 \\
& \frac{\delta y(L, t)}{\delta x}-\varphi(L, t)=0
\end{aligned}
$$

In order to obtain a steady-state solution to Eq. (2), we assume a solution of the form

$$
y(x, t)=y(x) \mathrm{e}^{j \omega t}
$$

which will yield the spatial complex amplitude distribution $Y(x)$. The shear force amplitude at the root of the cantilever is then

$$
V(x, t)=\chi A G\left(\varphi-\frac{\delta y}{\delta x}\right)
$$

The Eq. (2), subjected to boundary conditions Eq. (3), using expression Eq. (5) gives a shear force amplitude of

$$
V_{0}=\left(U_{1}+U_{2}\right) W_{0}
$$

with

$$
U_{1}=\frac{A T^{4}\left[\cos \left(\alpha_{1} L\right) \sinh \left(\alpha_{2} L\right) \alpha_{1} T^{2}-\sin \left(\alpha_{1} L\right) \cosh \left(\alpha_{2} L\right) \alpha_{2} T^{2}\right] B_{1}}{\left(\alpha_{2}^{2} \alpha_{1}^{2} \chi^{2} G^{2} Q\right)}
$$

where $B_{1}=\left(\alpha_{1}^{2}+\alpha_{2}^{2}\right) \rho^{3} \lambda^{6} \Omega_{1}^{6}$,

and

$$
U_{2}=\frac{A \rho^{2} T^{4}\left[-\cos \left(\alpha_{1} L\right) \sinh \left(\alpha_{2} L\right) \alpha_{1}^{3}-\sin \left(\alpha_{1} L\right) \cosh \left(\alpha_{2} L\right) \alpha_{2}^{3}\right] B_{2}}{\left(\alpha_{1}^{2} \alpha_{2}^{2} \chi G Q\right)}
$$

where $B_{2}=\left(\alpha_{1}^{2}+\alpha_{2}^{2}\right) \lambda^{4} \Omega_{1}^{4}$,

and 


$$
\begin{aligned}
Q= & Q_{1}+Q_{2}+Q_{3} \\
Q_{1}= & -\frac{T^{6} \Omega_{1}^{6} \lambda^{6} \rho^{3}}{\left(\chi^{3} G^{3} \alpha_{1}^{2} \alpha_{2}^{2}\right)}\left[-\alpha_{2}^{2} \sin \left(\alpha_{1} L\right) \sinh \left(\alpha_{2} L\right)-2 \alpha_{1} \alpha_{2}+\alpha_{1}^{2} \sin \left(\alpha_{1} L\right) \sinh \left(\alpha_{2} L\right)\right. \\
& \left.+2 \alpha_{1} \alpha_{2} \cos \left(\alpha_{1} L\right) \cosh \left(\alpha_{2} L\right)\right]
\end{aligned}
$$

and

$$
\begin{aligned}
Q_{2}= & \frac{T^{4} \Omega_{1}^{4} \lambda^{4} \rho^{2}}{\left(\alpha_{1}^{2} \alpha_{2}^{2} \chi^{2} G^{2}\right)}\left[2 \alpha_{1} \alpha_{2}^{3} \cos \left(\alpha_{1} L\right) \cosh \left(\alpha_{2} L\right)+2 \alpha_{1}^{3} \alpha_{2}-2 \alpha_{1} \alpha_{2}^{3}-\alpha_{1}^{4} \sin \left(\alpha_{1} L\right) \sinh \left(\alpha_{2} L\right)\right. \\
& \left.-2 \alpha_{1}^{3} \alpha_{2} \cos \left(\alpha_{1} L\right) \cosh \left(\alpha_{2} L\right)+2 \alpha_{1}^{2} \alpha_{2}^{2} \sin \left(\alpha_{1} L\right) \sinh \left(\alpha_{2} L\right)-\alpha_{2}^{4} \sin \left(\alpha_{1} L\right) \sinh \left(\alpha_{2} L\right)\right]
\end{aligned}
$$

and

$$
\begin{aligned}
Q_{3}= & -\frac{\rho T^{2} \Omega_{1}^{2} \lambda^{2}}{\alpha_{1}^{2} \alpha_{2}^{2} \chi G}\left[\alpha_{1} \alpha_{2}^{5} \cos \left(\alpha_{1} L\right) \cosh \left(\alpha_{2} L\right)-\sin \left(\alpha_{1} L\right) \alpha_{1}^{4} \alpha_{2}^{2} \sinh \left(\alpha_{2} L\right)\right. \\
& \left.+\alpha_{1}^{2} \sin \left(\alpha_{1} L\right) \sinh \left(\alpha_{2} L\right) \alpha_{2}^{4}+\alpha_{2} \alpha_{1}^{5} \cos \left(\alpha_{1} L\right) \cosh \left(\alpha_{2} L\right)+2 \alpha_{1}^{3} \alpha_{2}^{3}\right]
\end{aligned}
$$

The steady-state solution to Eq. (1) is

$$
w(x, t)=W_{0} e^{j \omega t}
$$

where $W_{0}$ is the complex amplitude, and the following equation must hold

$$
\left(-\lambda^{2}+1\right) W_{0}-\frac{P_{0}}{k}-\frac{2 V_{0}}{k}=0 .
$$

Note that the second forcing term on the right side of Eq. (11) is a function of the complex vibratory amplitude of the mass $W_{0}$. Solving Eq. (12) for complex amplitude $W_{0}$ as a function of the external forcing function amplitude $P_{0}$ one obtains

$$
W_{0}=\frac{C}{D}
$$

and

$$
C=-\frac{P_{0} \mu \alpha_{1}^{2} \alpha_{2}^{2} \chi^{2} G^{2} Q}{2}
$$

where

$$
\begin{aligned}
D= & \rho A \Omega_{1}^{2}\left[\left(L \alpha_{2}^{2} Q \alpha_{1}^{2} \lambda^{2}-\alpha_{2}^{2} Q \alpha_{1}^{2} L\right) G^{2} \chi^{2}+\left[-\cos \left(\alpha_{1} L\right) \sinh \left(\alpha_{2} L\right) \alpha_{1}^{5}+\right.\right. \\
& -\cos \left(\alpha_{1} L\right) \sinh \left(\alpha_{2} L\right) \alpha_{1}^{3} \alpha_{2}^{2}-\sin \left(\alpha_{1} L\right) \cosh \left(\alpha_{2} L\right) \alpha_{2}^{3} \alpha_{1}^{2}+ \\
& \left.-\sin \left(\alpha_{1} L\right) \cosh \left(\alpha_{2} L\right) \alpha_{2}^{5}\right] T^{4} \Omega_{1}^{2} \mu \rho \lambda^{4} G \chi+\left[\cos \left(\alpha_{1} L\right) \sinh \left(\alpha_{2} L\right) \alpha_{1}^{3}\right. \\
& +\cos \left(\alpha_{1} L\right) \sinh \left(\alpha_{2} L\right) \alpha_{1} \alpha_{2}^{2}-\sin \left(\alpha_{1} L\right) \cosh \left(\alpha_{2} L\right) \alpha_{2} \alpha_{1}^{2}+ \\
& \left.\left.-\sin \left(\alpha_{1} L\right) \cosh \left(\alpha_{2} L\right) \alpha_{2}^{3}\right] T^{6} \Omega_{1}^{4} \mu \rho^{2} \lambda^{6}\right]
\end{aligned}
$$

In order to get maximum benefit from the present analysis some non dimensional quantities are introduced. The tuning ratio $T$ is the ratio of the first natural frequency of the cantilever to the natural frequency of the main lumped parameter system

$$
T=\frac{\omega_{a}}{\Omega_{1}}
$$


The mass ratio $\mu$ is the ratio of the total absorber mass to that of mass $M$

$$
\mu=\frac{2 \rho A L}{M}
$$

The frequency ratio $\lambda$ is the ratio of the frequency to the natural frequency of the $k-M$ combination of

$$
\lambda=\frac{\omega}{\Omega_{1}}
$$

The static deflection of the main system is defined to be

$$
W_{s t}=\frac{P_{0}}{k}
$$

The dimensionless frequency response function is then

$$
\gamma=\left|\frac{W_{0}}{W_{s t}}\right|=\left|\frac{F}{H}\right|
$$

where

$$
F=\alpha_{1}^{2} \alpha_{2}^{2} \chi^{2} G^{2} Q L
$$

and

$$
\begin{aligned}
H= & \left(L \alpha_{2}^{2} Q \alpha_{1}^{2} \lambda^{2}-\alpha_{2}^{2} Q \alpha_{1}^{2} L\right) G^{2} \chi^{2}+T^{4} \Omega_{1}^{2} \mu \rho \lambda^{4} G \chi\left[-\cos \left(\alpha_{1} L\right) \sinh \left(\alpha_{2} L\right) \alpha_{1}^{5}\right. \\
& -\cos \left(\alpha_{1} L\right) \sinh \left(\alpha_{2} L\right) \alpha_{1}^{3} \alpha_{2}^{2}-\sin \left(\alpha_{1} L\right) \cosh \left(\alpha_{2} L\right) \alpha_{2}^{3} \alpha_{1}^{2} \\
& \left.-\sin \left(\alpha_{1} L\right) \cosh \left(\alpha_{2} L\right) \alpha_{2}^{5}\right]+T^{6} \Omega_{1}^{4} \mu \rho^{2} \lambda^{6}\left[\cos \left(\alpha_{1} L\right) \sinh \left(\alpha_{2} L\right) \alpha_{1}^{3}\right. \\
& +\cos \left(\alpha_{1} L\right) \sinh \left(\alpha_{2} L\right) \alpha_{1} \alpha_{2}^{2}-\sin \left(\alpha_{1} L\right) \cosh \left(\alpha_{2} L\right) \alpha_{2} \alpha_{1}^{2} \\
& \left.-\sin \left(\alpha_{1} L\right) \cosh \left(\alpha_{2} L\right) \alpha_{2}^{3}\right]
\end{aligned}
$$

with

$$
\alpha_{1}=\frac{1}{2} \lambda T \Omega_{1} \sqrt{2} \sqrt{\frac{\rho}{E}} \sqrt{\frac{E}{\chi G}+1+\sqrt{\left(\frac{E}{\chi G}-1\right)^{2}+\frac{4 A E}{\rho \lambda^{2} T^{2} \Omega_{1}^{2} I_{n}}}}
$$

and

$$
\alpha_{2}=\frac{1}{2} \lambda T \Omega_{1} \sqrt{2} \sqrt{\frac{\rho}{E}} \sqrt{-\frac{E}{\chi G}+1+\sqrt{\left(\frac{E}{\chi G}-1\right)^{2}+\frac{4 A E}{\rho \lambda^{2} T^{2} \Omega_{1}^{2} I_{n}}}}
$$

\section{Chebyshev's theorem}

In this section are recalled the main proposition of the Chebychev's theorem for the search of optimal parameters [9].

Let $f(x)$ be a continuous function in $[a, b]$ and $p(x)$ an approaching polynomial belonging to the class $P_{n}$ of polynomials with degree less or equal to $n$. As specified by Chebyshev's theorem, the best uniform approximation is attained when the condition

$$
\min \max |f(x)-p(x)|
$$

is fulfilled. The solution to the minimization problem stated by Eq. (25) is unique and it can be found considering the following theorem. Let $f(x)$ be a continuous function in $[a, b]$ and $p(x)$ the best uniform approaching polynomial of degree $n$. Moreover, let 


$$
E_{n}=\max |f(x)-p(x)|
$$

and

$$
\epsilon(x)=f(x)-p(x) .
$$

There are at least $(n+2)$ points $a \leqslant x_{1}<x_{2} \ldots<x_{n+2} \leqslant b$ where $\epsilon(x)$ assumes the values $\pm E_{n}$ and with alternating signs:

$$
\epsilon\left(x_{i}\right)= \pm E_{n}
$$

with $i=1,2, \ldots, n+2$ and

$$
\epsilon\left(x_{i}\right)=-\epsilon\left(x_{i+1}\right)
$$

with $i=1,2, \ldots, n+1$. Hence the best uniform approaching function is completely characterized by the property of equioscillation at $(n+2)$ points. This property is the basis of numerical schemes for computing the approximant polynomial. Applying the Chebyshev's theorem to the absorber design, the frequency response function around the input frequency, $\gamma(\lambda)$, is approximated by the straight line, $L_{1}$, which is the best uniform approximant of degree $n=1$. Thus the Chebyshev's theorem allows us to determine the optimal values of $\mu$ and $T$ such that the curve $\gamma(\lambda)$, has two peak values with minimum distance from a straight line $L_{1}$, where $L_{1}$ is initially unknown. The following system of non-linear algebraic equations can be written

$$
\begin{aligned}
& \left(\frac{\delta \gamma}{\delta \lambda}\right)_{\lambda=\lambda_{1}}=0 \\
& \left(\frac{\delta \gamma}{\delta \lambda}\right)_{\lambda=\lambda_{2}}=0 \\
& \left(\frac{\delta \gamma}{\delta \lambda}\right)_{\lambda=\lambda_{3}}=0 \\
& -\gamma\left(\lambda_{1}\right)+L_{1}+\Delta=0 \\
& -\gamma\left(\lambda_{2}\right)+L_{1}-\Delta=0 \\
& -\gamma\left(\lambda_{3}\right)+L_{1}+\Delta=0
\end{aligned}
$$

where $\Delta$ is the maximum deviation of the response curve from the value $L_{1}$. A curve attains a maximum or a minimum at frequency ratios $\lambda_{1}, \lambda_{2}$ and $\lambda_{3}$. Therefore, system Eq. (30) is composed of six equations with seven unknown variables $\lambda_{1}, \lambda_{2}, \lambda_{3}, \mu, T, L_{1}$ and $\Delta$. Solving the system of non-linear equation for different and prescribed values of $\mu$, it can be computed the numerical values of the optimal parameters.

\section{Numerical example}

Considering a system where the length of the beam is kept constant, the optimized frequency response function of the main mass is obtained making use of the design charts presented in Figs 4 and 5. In particular, in Fig. 4 is shown the graph which relates the optimal values of mass ratio as a function of the main mass $M$. In Fig. 5 is reported the graph of the optimal tuning ratio $T$ as a function of $\mu_{\mathrm{opt}}$. Thus once known the value of $\mu_{\mathrm{opt}}$ from the previous step, is possible to obtain also the optimal value of tuning ratio $T$. Using the Eq. (20) with both of the optimal values previously obtained, it is possible to define the final behavior of the frequency response function of the main mass. 


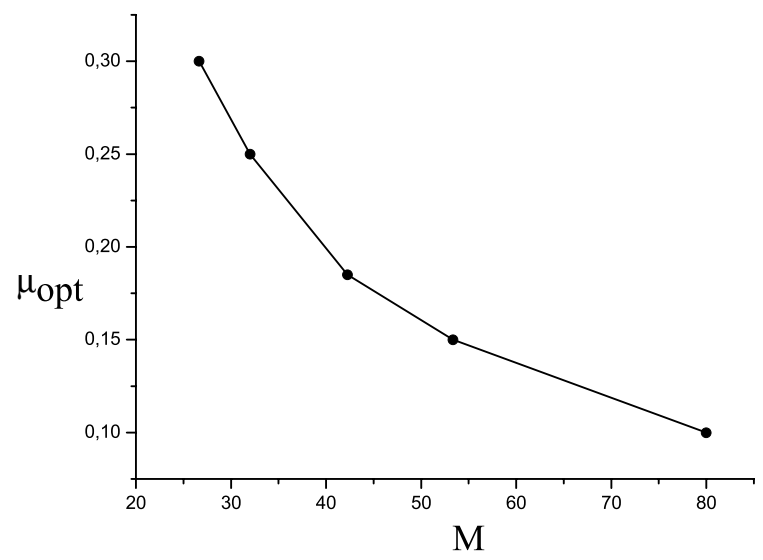

Fig. 4. Optimal values of mass ratio.

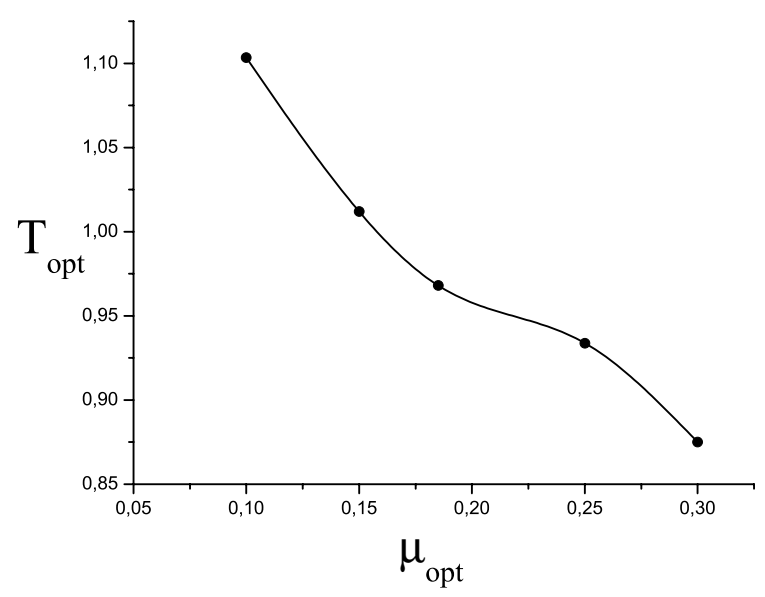

Fig. 5. Optimal tuning ratio.

The procedure previously discussed has been applied to a main mass of a primary system whose vibration needs to be controlled. The value of main mass is $M=42.243 \mathrm{~kg}$ and the vibration absorber is a uniform beam with the following pertinent specifications: $L=1 \mathrm{~m}, A=5 \cdot 10^{-4} \mathrm{~m}^{2}, E=2 \cdot 10^{11} \mathrm{~N} / \mathrm{m}^{2}, G=0.808 \cdot 10^{11} \mathrm{~N} / \mathrm{m}^{2}$, $\rho=8000 \mathrm{Kg} / \mathrm{m}^{3}, I_{n}=10^{-9} \mathrm{~m}^{4}$ and $\chi=0.833$. The optimal solution for the Timoshenko beam theory is $T_{\text {opt }}=0.968$ and $\mu_{\text {opt }}=0.185$.

In conclusion, the main advantages in using the present technique are:

- the two peaks of the main mass maximum displacement value are leveled;

- the use of Timoshenko beam theory guarantee a more faithful modeling of the beam dynamic behavior.

The optimal parameters, using the Euler - Bernoulli beam theory, are $T_{\mathrm{opt}}=0.865$ and $\mu_{\mathrm{opt}}=0.2$. These are not very far from the optimal solution obtained by means of Timoshenko theory. Figure 6 refers to a comparison between Euler - Bernoulli beam theory and Timoshenko beam theory with the computed optimal parameters $T_{\text {opt }}=0.968$ and $\mu_{\mathrm{opt}}=0.185$.

\section{Conclusions}

The authors have proposed a new method for the optimal design of a double-ended cantilever beam as a dynamic vibration absorber for a lumped-parameter single degree-of-freedom vibration system. The governing equation for 


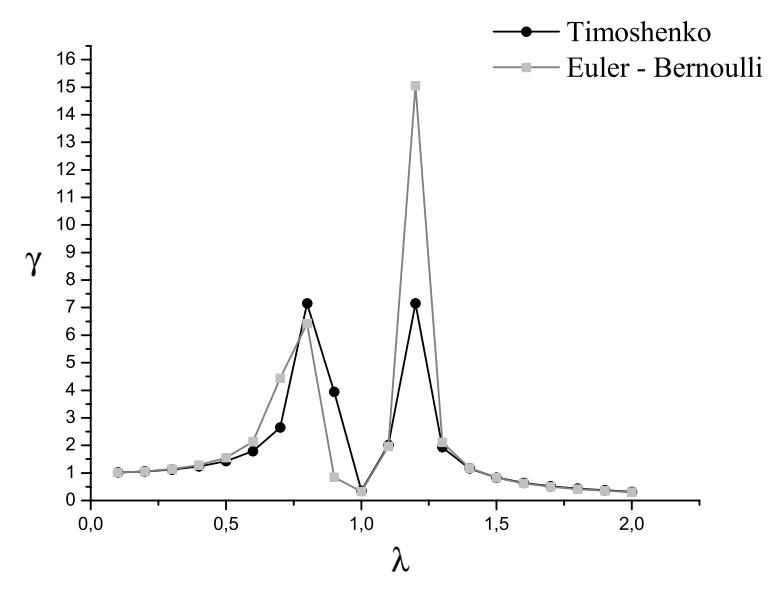

Fig. 6. Comparison between Timoshenko and Euler-Bernoulli theory.

the cantilever beam was developed under the assumption of Timoshenko bending theory. The Chebyshev's criterion was applied and the design charts for optimal beam-type absorber prepared. The results should be useful to designers of machine elements and structural systems.

\section{Acknowledgements}

The authors wish to acknowledge Prof. Ettore Pennestrì of University of Rome Tor Vergata for his helpful advice during the development of this work.

\section{NOMENCLATURE}

$A \quad$ area

E Young's modulus

G shear modulus

$I_{n} \quad$ area moment of inertia

$k \quad$ stiffness coefficient

$L \quad$ length

$L_{1} \quad$ straight line

$M \quad$ main mass

$P_{0} \quad$ external forcing function amplitude

$Q \quad$ solving equation of the system

$\dot{q}, \dot{w} \quad$ generalized velocities

$T \quad$ tuning ratio

$V \quad$ shear force

$W_{0} \quad$ complex amplitude

$W_{s t} \quad$ static deflection of the main system

$Y \quad$ spatial complex amplitude distribution

$\lambda \quad$ frequency ratio

$\mu \quad$ mass ratio

$\rho \quad$ mass density

$\chi \quad$ shear factor

$\Omega_{1} \quad$ natural frequency of the $k-M$ combination

$\omega \quad$ input frequency

$\omega_{a} \quad$ first natural frequency of the cantilever 


\section{References}

[1] J.K. Amdall and W.O. Jankovsky, Drive line vibration absorber, US Patent No. 3995513, December 7, 1976.

[2] G.A. Brewer, Dynamic vibration absorber, US Patent No. 4150588, April 24, 1979.

[3] A. Duckworth, Vibration isolator and shock absorber device, US Patent No. 4630809, December 23, 1986.

[4] H. Frahm, Device for damping vibrations of bodies, US Patent No. 989958, 1909.

[5] H. Ghoneim, On the Application of Optimum Damped Absorber to Vehicle Suspension 108 (March, 1986), 22-24.

[6] H. Ghoneim and S.M. Metwalli, Optimum Vehicle Suspension with a Damped Absorber, ASME Journal of Mechanism, Transmission and Automation in Design 106 (June, 1984), 148-155.

[7] C.C. Huang and T.C. Huang, Forced Vibrations of Viscoelastic Timoshenko Beams, Transactions of the ASME, Journal of Engineering for Industry (1976), 820-826.

[8] R.G. Jacquot and J.E. Foster, Optimal Cantilever Dynamic Vibration Absorbers, Journal of Engineering for Industry, Trans. ASME (1977), $138-141$.

[9] E. Pennestrì, An Application of Chebyshev's Min-Max Criterion to the Optimal Design of a Damped Dynamic Vibration Absorber, Journal of Sound and Vibration 217(4) (1998), 757-765.

[10] S.E. Randall, ASME, Journal of Mechanical Design 103, Optimum vibration absorbers for linear damped systems, 908-913.

[11] J.K. Vandiver, Mitome, Shuhei, Method and apparatus for absorbing dynamic forces on structures, US Patent No. 4226554, October 7, 1980 .

[12] P. Watts, On a method of reducing the rolling of ships at sea, Transaction of the Institutions of Naval Architects 24 (1983), 165-190. 

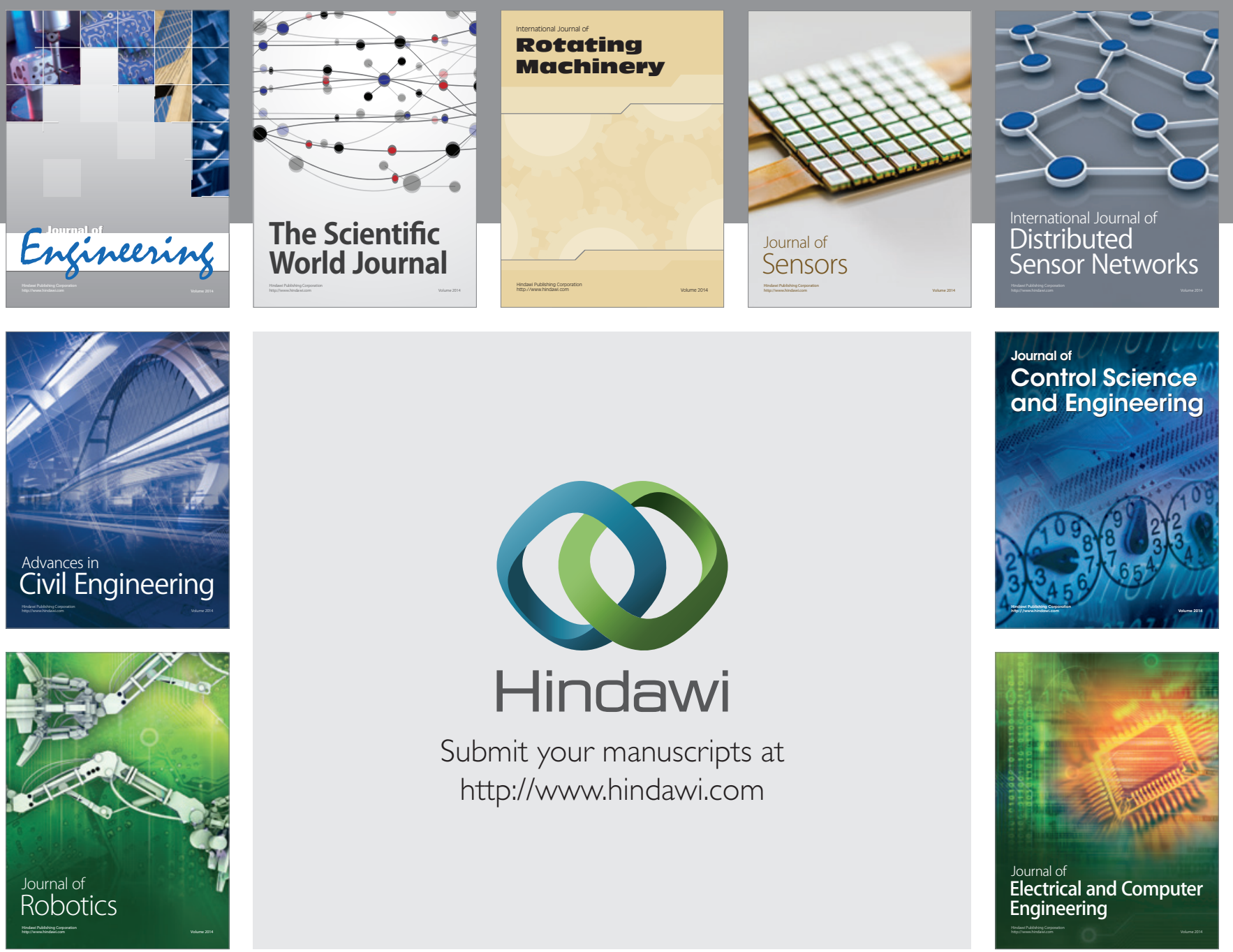

Submit your manuscripts at

http://www.hindawi.com
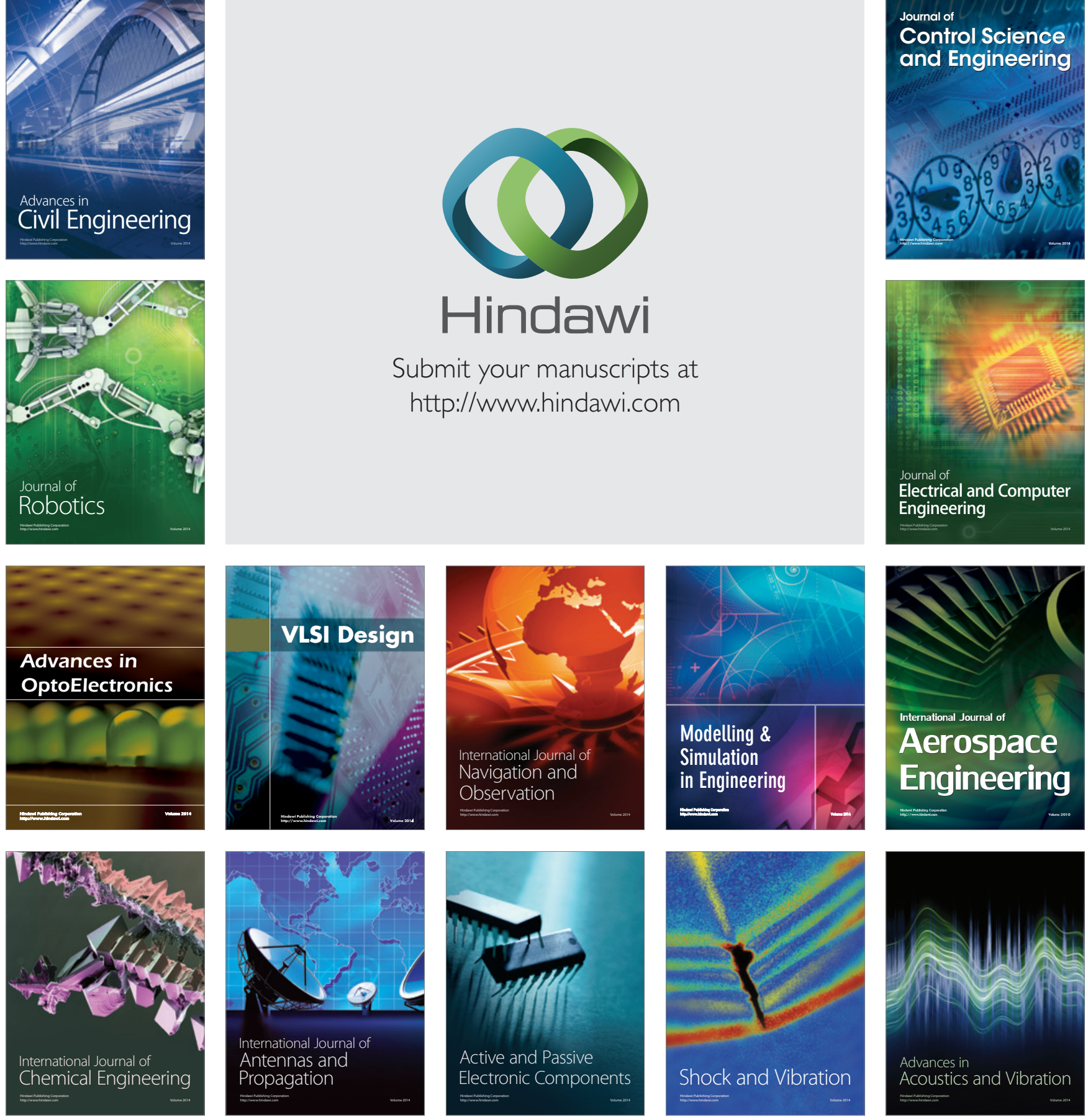